\title{
Stability analysis of hypersonic boundary layer flow over microporous surfaces
}

\author{
Viola Wartemann*, Heinrich Lüdeke ${ }^{\dagger}$ \\ DLR Institute for Aerodynamics and Flow Technology, Braunschweig, Germany \\ Neil D. Sandham ${ }^{\ddagger}$ \\ School of Engineering Sciences, University of Southampton, Southampton SO17 1BJ, U.K.
}

\begin{abstract}
Hypersonic boundary-layer-transition is dominated by so-called Mack modes, second mode instabilities that can be damped passively by acoustic absorptive coatings. Those coatings are realised in practise by porous walls. In the present paper a second mode stability analysis is performed for a boundary layer flow at Mach 6 over a smooth wall and various porous walls. The influence of the porosity, the radius and the thickness of the pores is investigated. For this study three different codes are used: The linear stability code SLST of the University of Southampton in comparison with the DLR linear stability code NOLOT and finally direct numerical simulations including the modelling of the pores through the wall. Good agreement for a smooth wall as well as for different porous wall cases is demonstrated.
\end{abstract}

\section{Introduction}

A typical challenge for re-entry vehicles during flight is an effective control of hypersonic transition and the laminar/turbulent state of the boundary layer. The state of the boundary layer is of high importance since skin friction drag and heat transfer rates in a turbulent boundary layer can be several times higher than those of a laminar one. A lot of different strategies are used to delay or prevent the transition process. In this work the manipulation of the transition is performed by the use of porous surfaces to influence the growth of the second mode in a passive way. The second mode or so called Mack mode ${ }^{1}$ is the dominant mode for the transition process at hypersonic Mach numbers.

Malmuth et al. ${ }^{2}$ have shown by inviscid linear stability theory a strong stabilisation effect of the second mode using a passive porous surface. In a continuation study Federov et al. ${ }^{3}$ confirmed the previous work by the use of viscous linear stability theory. Experiments of Rasheed ${ }^{4}$ with a $5^{\circ}$ half-angle sharp cone $\left(M a_{\infty} \sim 5-6\right)$ and blind cylindrical holes $(\varnothing \sim 60 \mu \mathrm{m})$ qualitatively confirmed the theoretical prediction. The working group around Federov performed further analyses and experiments. A good summary is given by Maslov et al. ${ }^{5}$. Two-dimensional direct numerical simulation studies that resolve the pores are presented by Bres et al. ${ }^{6,7}$, where the linear stability theory code was verified. A study of the effect by direct numerical simulations with a three-dimensional pore flow is presented by Sandham and Lüdeke. ${ }^{8}$

In this paper results of the DLR NOLOT code, which is a spatial linear stability code, are compared with the temporal / spatial linear stability code of the University of Southampton and direct numerical simulations by the DLR FLOWer code. ${ }^{8,9}$ Good agreement of all codes could be shown by a comparison for a Mach 6 boundary layer flow over a smooth wall as well as for different porous walls. Also a comparison between previous results of Federov ${ }^{3}$ with NOLOT will demonstrate good comparability. Additionally a

\footnotetext{
*Ph.D. Student, DLR, viola.wartemann@dlr.de

${ }^{\dagger}$ Research Scientist, DLR, heinrich.luedeke@dlr.de

${ }^{\ddagger}$ Professor, School of Engineering Sciences, n.sandham@soton.ac.uk, AIAA Senior Member
} 
numerical study with NOLOT for a Mach 7 blunt cone case, for which wind tunnel test are provided, is carried out.

\section{Numerical methods}

\section{A. Linear stability theory - LST}

\section{NOLOT - NOnLocal Transition analysis}

The NOLOT code, ${ }^{10}$ which is a spatial linear stability code, was developed in cooperation between DLR and FOI and can be used for local as well as non-local analyses. In this work the local linear spatial method is used which is a subset of the nonlocal stability equations. The equations are derived from the equations of conservation of mass, momentum and energy which govern the flow of a viscous, compressible, ideal gas, formulated in primitive variables. All flow and material quantities are decomposed into a steady laminar basic flow $\bar{q}$ and an unsteady disturbance flow $\tilde{q}$.

$$
q(x, y, z, t)=\bar{q}(x, y)+\tilde{q}(x, y, z, t)
$$

The disturbance $\tilde{q}$ is represented as a harmonic wave

$$
\tilde{q}(x, y, z, t)=\hat{q}(x, y) \exp [i(\alpha x+\beta z-\omega t)]
$$

with the complex-valued amplitude function $\hat{q}$. In the following the hat over a variable denotes an amplitude function. Since NOLOT is a spatial code the wavenumbers $\alpha$ and $\beta$ are complex quantities and the frequency $\omega$ is a real value. $-\alpha_{i}$ is the complex growth rate. The given boundary conditions in NOLOT for a smooth wall (at $y=0$ ) are:

$$
\hat{u}_{w}, \hat{v}_{w}, \hat{w}_{w}, \hat{T}_{w}=0 .
$$

The NOLOT code (without the porous surface boundary condition) is validated with the help of several test cases against published results, including DNS, PSE, multiple scales methods and LST. A good summary of the validation is given by Hein et al. ${ }^{10}$. For the treatment of porous walls, additional boundary conditions are implemented, representing the influence of the pores on the instabilities. These boundary conditions for porous walls are extracted from Federov et al. ${ }^{3}$, a complete derivation can be found in this reference. The conditions are written by:

$$
\hat{u}_{w}, \hat{v}_{w}=0, \quad \hat{w}_{w}=A \hat{p}_{w}, \quad \hat{T}_{w}=B \hat{p}_{w}
$$

with the admittance A:

$$
A=\frac{n}{Z_{0}} \tanh (\Lambda d)
$$

and with $Z_{0}$ :

$$
Z_{0}=\sqrt{\frac{\frac{i \omega J_{0}\left(k_{v}\right)}{T_{w} J_{2}\left(k_{v}\right)}}{-i \omega M a^{2}\left[\gamma+(\gamma-1) \frac{J_{2}\left(k_{t}\right)}{J_{0}\left(k_{t}\right)}\right]}} .
$$

$J_{0}$ and $J_{2}$ are Bessel functions of the complex arguments $k_{v}$ and $k_{t}$ :

$$
k_{v}=r \sqrt{\frac{i \omega \rho_{w} R e}{\mu_{w}}} \quad k_{t}=k_{v} \sqrt{\operatorname{Pr}}
$$

The investigated pores are equally spaced cylindrical blind holes with a depth $d$ and a radius $r$, which are normalised with the displacement thickness $\delta$, and the porosity $n$. The dimensionless flow quantities are normalised by the values of the boundary layer edge. $\omega$ represents the dimensionless angular frequency. The thermal admittance, which can be defined in a similar way, was found to have a marginal effect for the investigated cases $(<1 \%)^{3}$ and is neglected in the present work.

\section{Southampton SLST code}

The linear stability code of the University of Southampton can be used for temporal as well as spatial analyses in contrast to the spatial treatment in NOLOT. The code solves the compressible Orr-Sommerfeld equations for the growth of small disturbances superimposed on the prescribed base flow. The Orr-Sommerfeld 
results presented here are based on a derivation from the conservative Navier-Stokes equations. The wave functions are the same as described in equation 2. If the code is used as a temporal stability solver, because of the comparison with the temporal DNS, the wavenumbers $\alpha$ and $\beta$ are real quantities and the frequency $\omega$ is a complex value. The imaginary part $\omega_{i}$ is the growth rate which is finally the quantity of interest. A direct matrix solution method is used, with derivatives computed by a mapped Chebyshev method using a minimum of 150 collocation points. The code was validated by comparison with a number of sources in the literature, including Malik. ${ }^{12}$ The boundary condition formulation for the porous walls ${ }^{3}$ (without the thermal admittance) are described in section A1.

\section{B. Direct numerical simulation (DNS) with FLOWer}

For the DNS calculations a high-order variant of the DLR FLOWer code is used. The basic FLOWer code solves the compressible Reynolds-averaged Navier Stokes equations, which are written in the conservation law form, for a flow of a perfect gas on block-structured grids with second order finite volume techniques and cell-centred or cell vertex variables. The high-order version uses fourth-order central differencing based on standard compact finite differences in a cell-centred formulation, stabilised by high-order compact filters, applied at the end of each time step. ${ }^{11}$ The DNS is initialised with an artificial disturbance at $t=0$ and the eigenmode is allowed to develop naturally within the calculation. The initial disturbance, which is a rough approximation of a second mode eigenfunction, is given by:

$$
v=0.0001 \exp \left[-4(y-0.8)^{2}\right] \sin \left(2 \pi x / L_{x}\right)
$$

The pores are described as rectangular pores in a way that the grids match at the interface, removing interpolation as a possible source of error.

\section{Validation of NOLOT by test cases of Federov et al.}

For the first validation of the stability code, test cases of Federov et al. ${ }^{3}$ with the following base flow parameters are used: $M_{\infty}=6$, Prandtl number $\operatorname{Pr}=0.72$, ratio of specific heats $\gamma=1.4$, an adiabatic wall boundary condition and different Reynolds numbers. The comparison cases take a porosity of 0.5 and a pore radius of 0.05 , which is normalized with the displacement thickness. The pore depth goes to infinity. For the wall normal direction 401 points are set for the NOLOT discretisation.
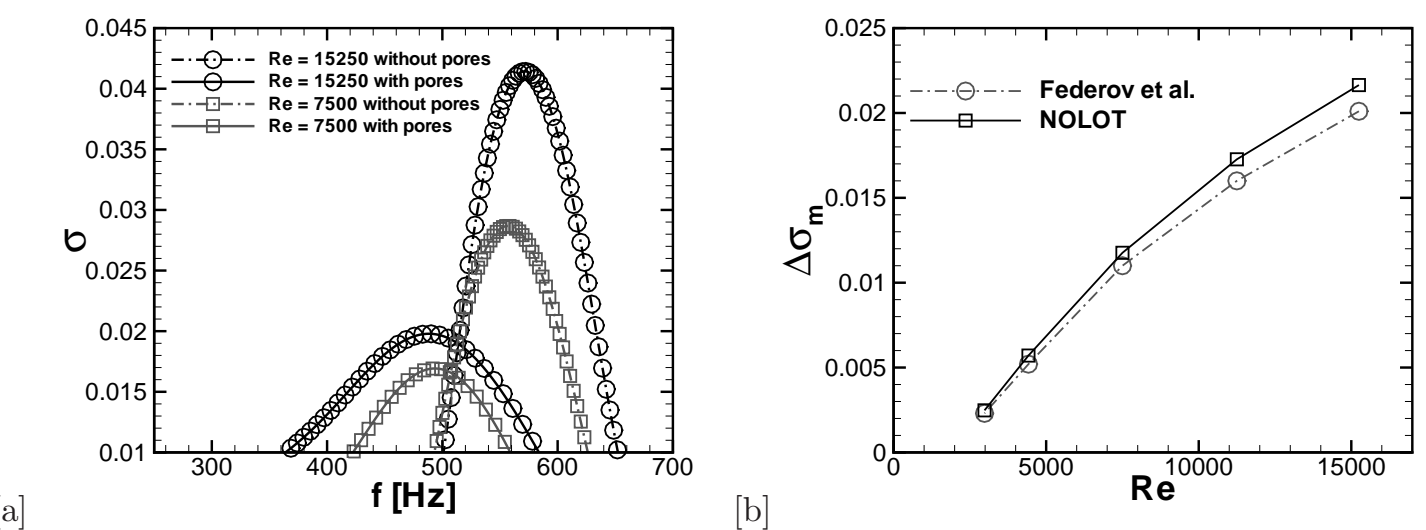

Figure 1. (a) Growth rates against frequency for different Reynolds numbers with and without pores (b) Comparison of the relative growth rate between NOLOT and stability results from Federov ${ }^{3}$ with a porous surface

Figure 1a shows the NOLOT results for two different Reynolds numbers. A strong reduction of the growth rate of the second mode over a wide frequency band can be seen. In Figure 1b the difference between the maximum growth rate of the smooth wall and the porous wall calculated with NOLOT is compared with the results from Federov. The average relative difference is less then $8 \%$, which demonstrates a good agreement for the two different codes with different grids and only approximately equally conditions. Due to these uncertainties in the input data a second validation is given against the Southampton LST code with 
identical base flow parameters and grids in comparison with the DNS for a smooth wall as well as different porous walls.

\section{Comparison of LST with DNS for the smooth wall test case}

The base flow parameters for the smooth wall case for all three codes are: $M a_{\infty}=6$ at a Reynolds number of $\operatorname{Re}=20000$, a Prandtl number $\operatorname{Pr}=0.72$, the ratio of specific heats $\gamma=1.4$ and an adiabatic wall boundary condition. Viscosity $\mu$ is prescribed by Sutherland's law with a constant of $110.4 \mathrm{~K}$ and a reference temperature of $216.65 \mathrm{~K}$, leading to a wall temperature of $1522.44 \mathrm{~K}$.

For the wall normal grid distribution a stretching function is used which places most points near the wall in an analytical way. The distribution is given by a sinh function with an iteratively determined stretching factor. In the wall normal direction 401 points are set for all codes in the same way. For the DNS a 2D grid with 32 equally spaced points in streamwise direction is generated. All the coordinates are normalised by the displacement thickness $\delta$.

In figure 2 stability diagrams for LST are shown. The SLST code is used in this case in the temporal

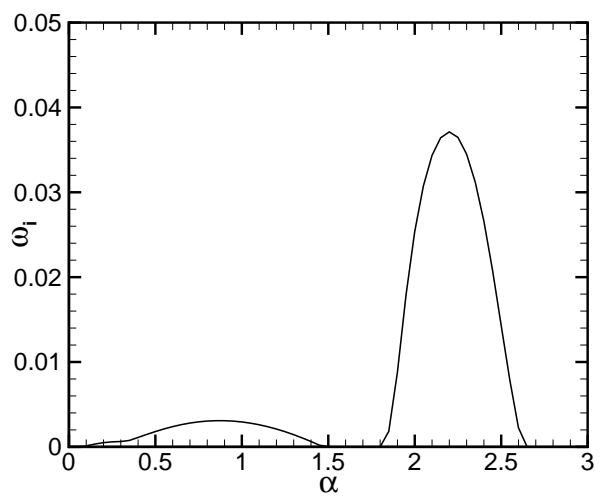

[a]

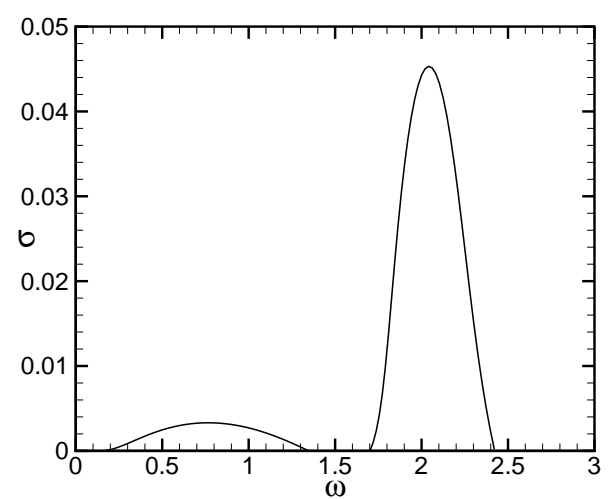

[b]

Figure 2. Smooth wall case: (a) Temporal growth rate against wavenumber $\alpha$ (b) Spacial growth rate against dimensionless frequency $\omega$

mode to provide comparability with the DNS, thus the temporal growth rate $\omega_{i}$ is plotted as a function of the wavenumber $\alpha$ for the SLST code, while for the spatial NOLOT code the growth rate is $\sigma$ as a function of the dimensionless frequency $\omega$. For a comparison between $\sigma$ and $\omega_{i}$ obviously a transformation is necessary, which is well known as a Gaster transformation ${ }^{1}$ :

$$
\sigma_{\text {spatial }}=\frac{\omega_{i, \text { temporal }}}{c_{g r, \text { temporal }}}
$$

with the group velocity $c_{g r}$

$$
c_{g r, \text { temporal }}=\frac{\Delta \omega_{r, \text { temporal }}}{\Delta \alpha_{\text {temporal }}} .
$$

The most unstable mode is the second mode with a maximum temporal growth rate of 0.037 near $\alpha=2.2$. By using Gaster transformation a maximum spatial growth rate of 0.0453 can be calculated from temporal results. The maximum value predicted by NOLOT is marginally larger as shown in figure $2 \mathrm{~b}$. Figure 3 demonstrates good agreement of all three codes. In Figure 3a the velocity amplitude function is plotted against the wall normal direction and figure 3b compares the stability and DNS results of the disturbance amplitude. Since the DNS is initialised by an artificial disturbance at $t=0$ (see equation (8)) the simulation needs a small number of time steps until the unstable mode has emerged. The relative difference between amplification factors from NOLOT and DNS has a value of $1.3 \%$ and between SLST and DNS of 0.3\%, so an excellent agreement of all different approaches could be shown.

\section{E. Comparison of LST with DNS for various porous test cases}

The flow conditions for these cases are the same as those of the smooth wall case. Also the grids are the same for the LST codes. For the DNS simulation a refined 2D grid with 64 points per pore spacing is 

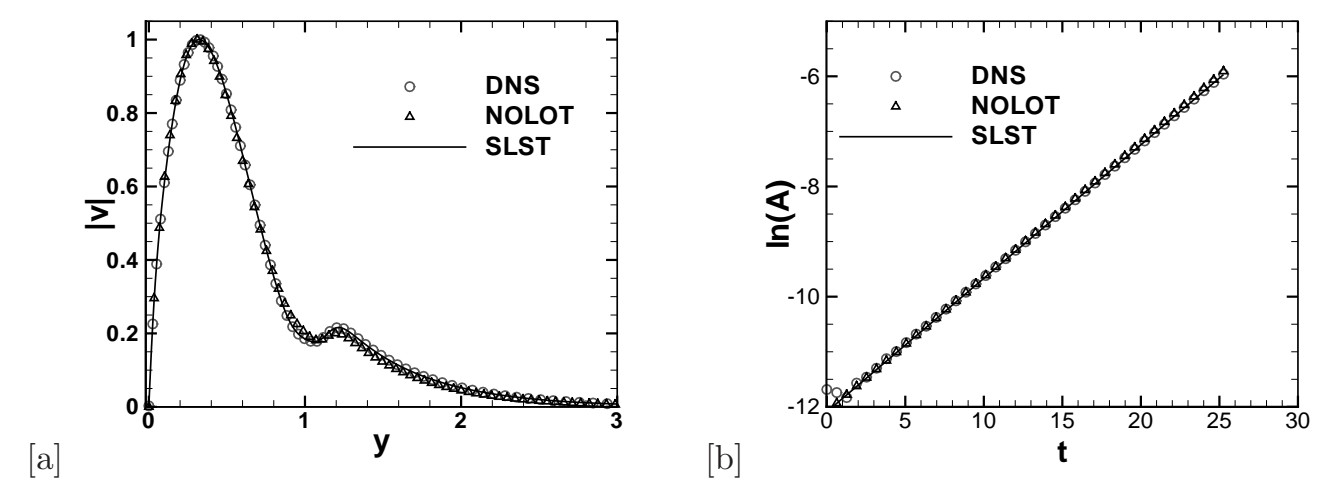

Figure 3. Smooth wall case: (a) Comparison of the amplitude functions (b) DNS of disturbance amplitude and LST growth rate

generated. The number of points for $y>0$ is again 401 and the additional points within the pores $(y<0)$ depend on the depth of the pores. As an example for the pore depth of $d=1$ a number of 168 points has been chosen. The pore depth and the coordinates are normalised by the displacement thickness. Since the simulations with the DNS are two-dimensional the porous surface is reduced to spanwise grooves with parallel sides.

In the following a detailed comparison of all three codes is given by using the test case of figure 4, which shows DNS results from Mack modes developing on a smooth surface in comparison with a porous surface with pores of depth 1 , a pore radius of 0.046875 and porosity of $n=0.25$. This corresponds to 16 pores for the DNS. On the left side it can be seen the normal velocity over a smooth wall and the right side shows the reduction of the Mack mode amplitude due to the pore effect of absorbing a part of the disturbance energy. This is visible by comparing the legends of both figures: The values with pores are one order of magnitude smaller.
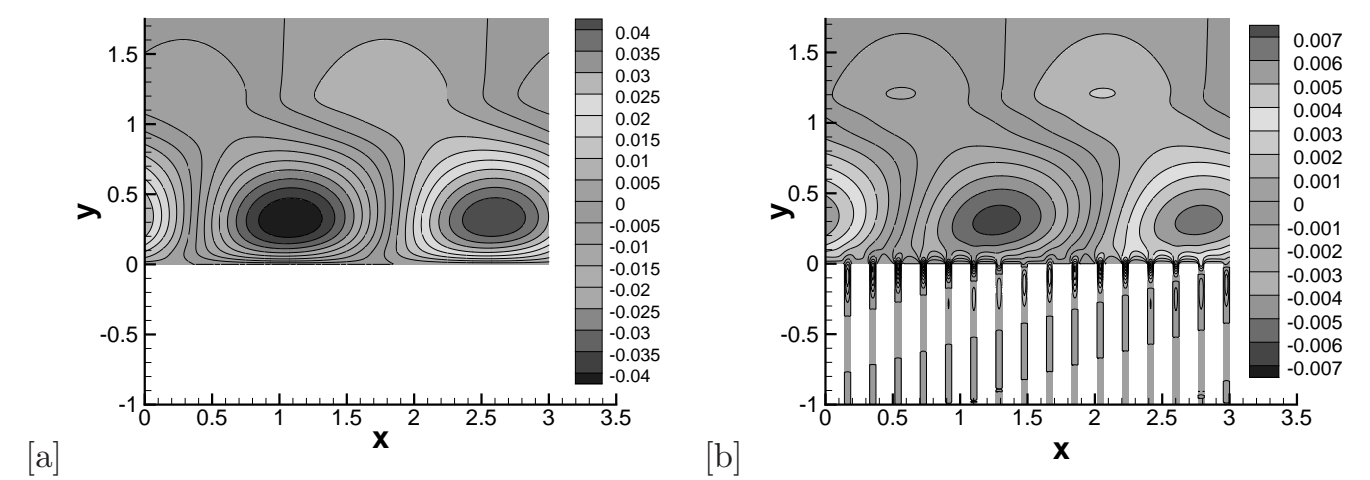

Figure 4. DNS (contours of $v$ ): (a) Mack mode developing above a smooth wall and (b) a porous surface ( $d=1$ and $r=0.046875)$ at the same time step

The stability diagrams from the LST results for the second mode are show in figure 5. By using the Gaster Transformation, the maximum spatial SLST growth rate, calculated by the SLST code, is 0.025866 . Again, as already shown by the smooth wall case, the growth rate predicted by NOLOT is marginally higher $(\sim 0.1 \%)$. In comparison to the smooth wall the maximum growth rate is reduced by about $40 \%$ (compare with figure 2).

For a quicker and more direct comparison of the LST codes without using approximative Gaster Transformation, this test case and also additional cases were spatially calculated by the SLST code.

As an additional difficulty former studies ${ }^{8}$ have shown a slight difference of the stability results on the formulation of the compressible Navier-Stokes equations, which can be given in conservative or primitive formulation (see appendix A). While normally the SLST code uses the conservative formulation of the Navier-Stokes equations, for the following study also results from the primitive formulation are shown. In 


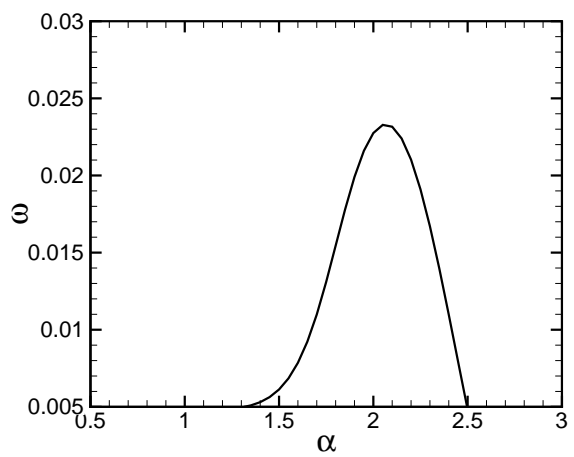

[a]

$\alpha$

[b]

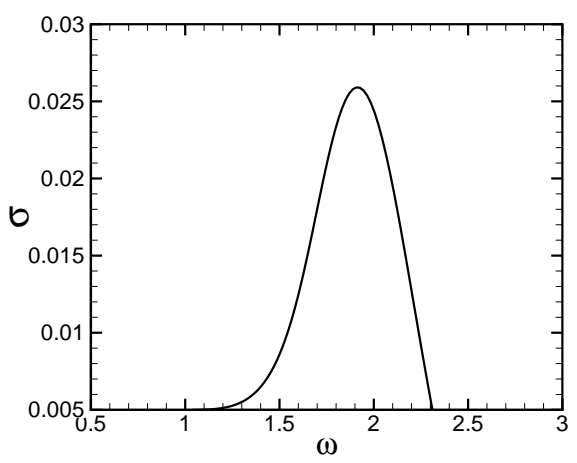

Figure 5. Porous wall case with $d=1$ and $r=0.046875$ : Growth rate against (a) wavenumber $\alpha$ and (b) dimensionless frequency $\omega$

the latter case nearly no differences (up to the $5^{\text {th }}$ decimal) between the calculated growth rates and NOLOT calculations are observed due to the primitive formulation of NOLOT. The first row of table 1 shows the different growth rates $\alpha_{i}$ for a dimensionless frequency of 2.0. The relative difference in this case is between the two SLST growth rates $0.33 \%$ and between the growth rates of NOLOT and SLST (primitive formulation) less than $0.05 \%$. The $0.05 \%$ demonstrates the excellent agreement between the codes using identical formulations. To emphasize this agreement additional test cases are calculated from NOLOT and SLST (see table 1) by using also a dimensionless frequency of 2.0 and a porosity of 0.25 .

\begin{tabular}{|r|c|c|c|c|c|}
\hline$R e$ & $d$ & $r$ & $\begin{array}{c}\alpha_{i} \text { NOLOT } \\
\text { primitive formulation }\end{array}$ & $\begin{array}{c}\alpha_{i} \text { SLST } \\
\text { primitive formulation }\end{array}$ & $\begin{array}{c}\alpha_{i \text { SLST }} \\
\text { conservative formulation }\end{array}$ \\
\hline 20000 & 1.0 & 0.046875 & 0.02443 & 0.02442 & 0.02453 \\
20000 & 0.3 & 0.046875 & 0.02311 & 0.02310 & 0.02321 \\
20000 & 1.0 & 0.093750 & 0.01870 & 0.01870 & 0.01881 \\
20000 & 0.3 & 0.093750 & 0.00897 & 0.00898 & 0.00901 \\
6000 & 1.0 & 0.046875 & 0.01385 & 0.01385 & 0.01413 \\
\hline
\end{tabular}

Table 1. Comparison of the LST growth rates for various test cases

For the test case with $d=1$ and $r=0.046875$ figure 6 again shows a comparison between eigenfunctions and amplitude growth calculated from DNS and LST as already shown for the smooth case (see figure 3 ). The velocity amplitude functions (figure 6a) and the amplitude growth rates in figure 6b show good agreement. The growth rates of the different LST codes are nearly identical and the relative difference between the gradient of the DNS and the LST is about $6 \%$.

A detailed parameter study, including the just listed case, has been performed at the same flow conditions and porosity $(n=0.25)$ as the previous case has been carried out, by varying the pore depth and using two different pore radii $(r=0.046875$ and $r=0.09375)$ for DNS and SLST. For NOLOT an additional pore radius of $r=0.0625$ was taken into account to get a better overview of the influence of the radius. The pore radius corresponds to 16,8 and 12 pores for the DNS. The wavenumber $\alpha$ is set to the constant value of $2 \pi / 3$ for the whole parameter study. It is also possible to compare the maximum growth rates, which mean a variation of the wavenumber $\alpha$ at different pore depths, illustrates in figure 7 . The calculations are performed by NOLOT at $r=0.09375$ ( 8 pores) for varying pore depths. Figure 7 shows the growth rate as a function of the real part of the wavenumber $\alpha$. It can be seen that the wavenumber of the maximum growth rate changes with the pore depth down to a depth of about 0.4 , where the growth rate levels out to a nearly constant value. The functions of the pore depth of $0.4,0.5$ and 1.0 , which illustrated this, are labelled as black lines and show very small differences.

The same tendency can be found in figure 8 , where the growth rate is plotted as a function of the pore depth. 

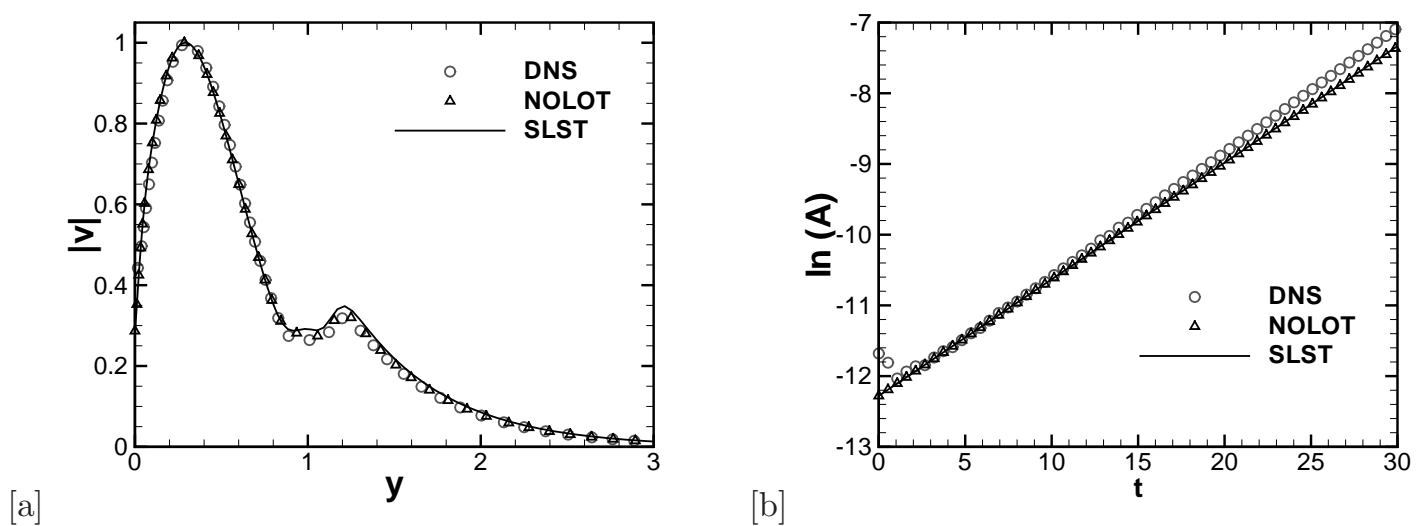

Figure 6. Porous wall case with $d=1$ and $r=0.046875$ : (a) Comparison of the amplitude functions (b) DNS of disturbance amplitude and LST growth rates

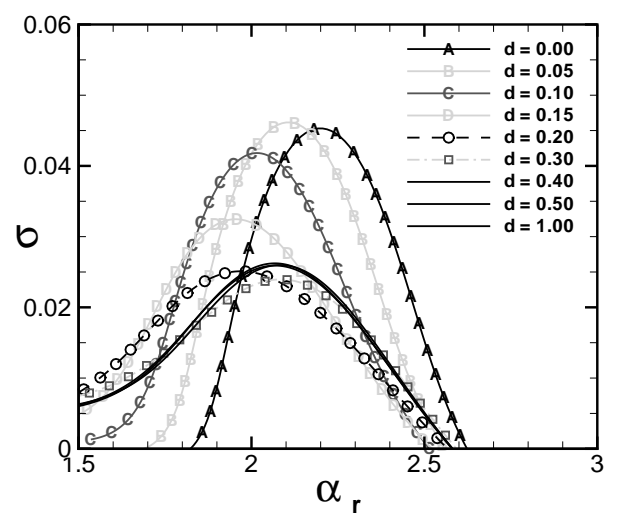

Figure 7. NOLOT results of the test case with $r=0.09375$ : Growth rate as a function of the real part of the wavenumber $\alpha_{r}$
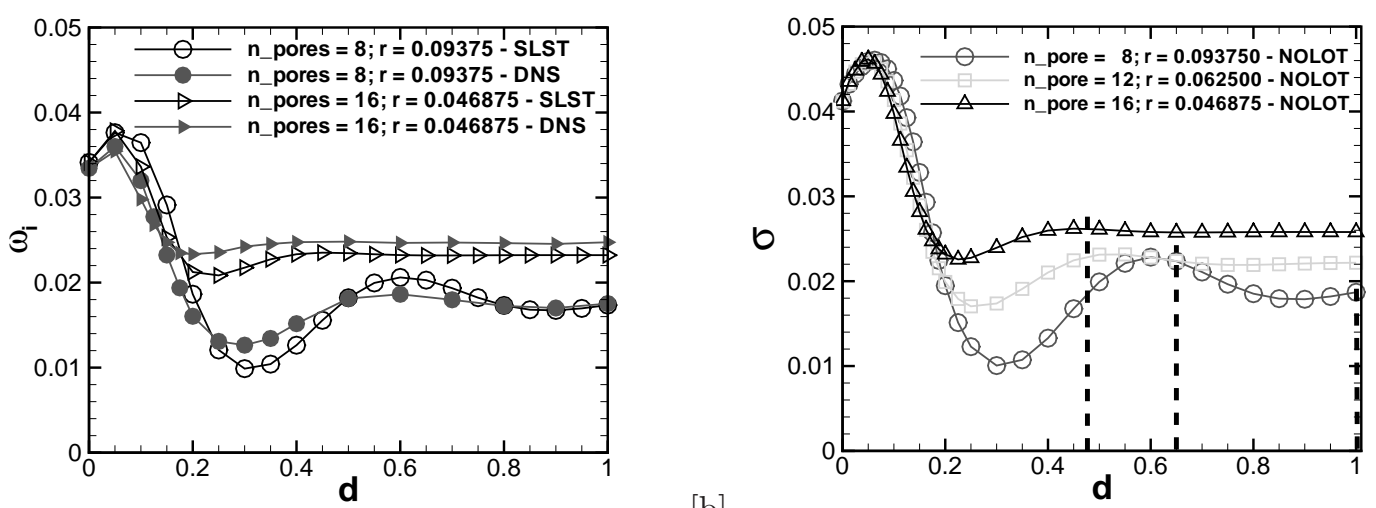

$[a]$

Figure 8. Comparison of all three codes: Growth rate against pore depth: (a) temporal results (b) spatial results

The good agreement between the SLST and NOLOT code was outlined in detail above and contains some of these test cases, thus here only the differences between temporal SLST and DNS are considered, which is shown in figure 8a. It should be noted that in comparison of previously published work ${ }^{8}$ an error in the SLST porous wall boundary implementation has been corrected, with a small improvement in the 
agreement with the DNS. For the test case with 8 pores $\left(r_{\text {pore }}=0.09375\right)$ the pore depth has a stronger effect calculated by SLST code than by the DNS. For deeper pores an end of the resulting growth rates appears. In the following the pore depth for which the variation of the growth rate is less than $2 \%$, is denoted as the limit-pore depth and the corresponding growth rate as the limit-growth rate. For the test case with 8 pores the limit-growth rate has a value of about 0.017. As a result the growth rate reduction between the smooth wall case $\left(\omega_{i}=0.033\right.$ at $\left.d=0\right)$ and limit-growth rate is about $50 \%$ (with LST a little bit higher than with DNS). For 16 pores where the radius is reduced by a factor of 2 the calculated growth rate at $d=1$ is found within a range of 0.24 by both approaches. This leads to a growth rate reduction of $\sim 30 \%$. For 16 pores at first glance a parallel offset seems to be obvious. This is due to the fact that in this case a second maximum does not exist and the end value difference is higher than for the 8 pore case. The relative differences are in the same range for both cases and the tendency of the growth rates, predicted by SLST and DNS, are identical. The cause of the difference can be found in the different modelling of the pores: The boundary condition in the SLST code derived from the approach of cylindrical pores while for the DNS the pores are modelled by $2 \mathrm{D}$ grooves. From previous studies of Sandham et al. ${ }^{8}$ a negative influence of the growth rate can be excluded for the $2 \mathrm{D}$ simplification. Sandham et al. ${ }^{8}$ shows that a reduction from $3 \mathrm{D}$ square pores to $2 \mathrm{D}$ grooves produces only small differences of the growth rate of about $6 \%$. Comparability of cylindrical and square pores is obtained by using the definition of a hydraulic diameter. ${ }^{13}$ However the trend of the functions is identical, the limit-pore depth is nearly the same and for these cases the limit-growth rate has a maximal relative difference below $7 \%$. This is a good agreement for two codes (SLST and DNS) with completely different approaches.

To confirm these statements the spatial NOLOT results are shown in figure $8 \mathrm{~b}$, where the spatial growth rates are given as a function of the pore depth with an added variation of the radius. The additional pore radius case confirmed that the growth rate approaches the limit-growth rate and that pores with larger radius have to be deeper to reach this limit-growth rate. Federov ${ }^{3}$ described for an example test case that a pore depth of about 5 diameters is sufficient for the appropriate damping rate, which corresponds to our results. The approximate pore depth of the limit-growth rates are extracted from figure $8 \mathrm{~b}$ (labelled with vertical dashed lines) and are listed in table 2 in column 4 . The limit-pore depth of row one $(r=0.09375)$ is described with $>1$ due to the calculated values go to this value. As visible in figure $8 \mathrm{~b}$ the limit-pore depth is higher than 1 (The here given definition of the limit-pore depth was: The variation of the growth rate is less than $2 \%$ ). The pore diameter multiplied with 5 to connect to the test case of Federov is shown in column 3. As visible the values of the limit-pore depths are in the same range.

\begin{tabular}{|c|c|c|c|}
\hline number of pores & pore radius & pore diameter $\cdot 5$ & limit-pore depth \\
\hline 8 & 0.093750 & 0.93750 & $>1$ \\
12 & 0.062500 & 0.62500 & 0.65 \\
16 & 0.046875 & 0.46875 & 0.48 \\
\hline
\end{tabular}

Table 2. Pore depth of the limit-growth rate

\section{F. Preview of planned wind tunnel tests}

In addition to the numerical investigation different wind tunnel tests are planned to complete the study. This is a part of the IMENS-3C project (Integrated Multi-disciplinary design of Hot Structures). For this project a large, detailed series of measurements will be performed in two different wind tunnels. The first facility is the high enthalpy wind tunnel at the DLR in Göttingen (HEG) and the second the hypersonic tunnel of the DLR in Köln-Porz $(\mathrm{H} 2 \mathrm{~K})$. For the HEG a $7^{\circ}$ blunt cone with a nose radius of $2.5 \mathrm{~mm}$ and for the $\mathrm{H} 2 \mathrm{~K}$ a $3^{\circ}$ blunt cone with various nose radii $(1 \mathrm{~mm}, 5 \mathrm{~mm}, 10 \mathrm{~mm}$ and $15 \mathrm{~mm}$ ) with and without pore surfaces are planned. Similar free steam conditions (with $M a>6$ ) will be performed. Figure 9 shows some typical numerical results of NOLOT for one planned free stream condition of the H2K: $M a=7$, $R e_{u}=3.7 \cdot 10^{6} / \mathrm{m}, T=54 \mathrm{~K}, p=242 \mathrm{~N} / \mathrm{m}^{2}$. On the left side the maximum growth rate as a function of the pore depth is shown for a constant porosity of 0.2 and a constant radius of $r=0.25 \mu \mathrm{m}$. The maximum growth rate reaches the limit-growth rate of 6.7 (reduction of $\sim 50 \%$ ) at a pore depth of about $300 \mu \mathrm{m}$. Figure 9b illustrates the connection between porosity and growth rate for a constant radius of $r=0.25 \mu \mathrm{m}$ where the pore depth is set to infinity. At a porosity of only $n=0.05$ the growth rate is reduced by $20 \%$ 
and at $n=0.25$ by $50 \%$. Further investigations are still running to identify suitable pore parameters for the experiments. With NOLOT the possibility is given to do this in a very fast way.
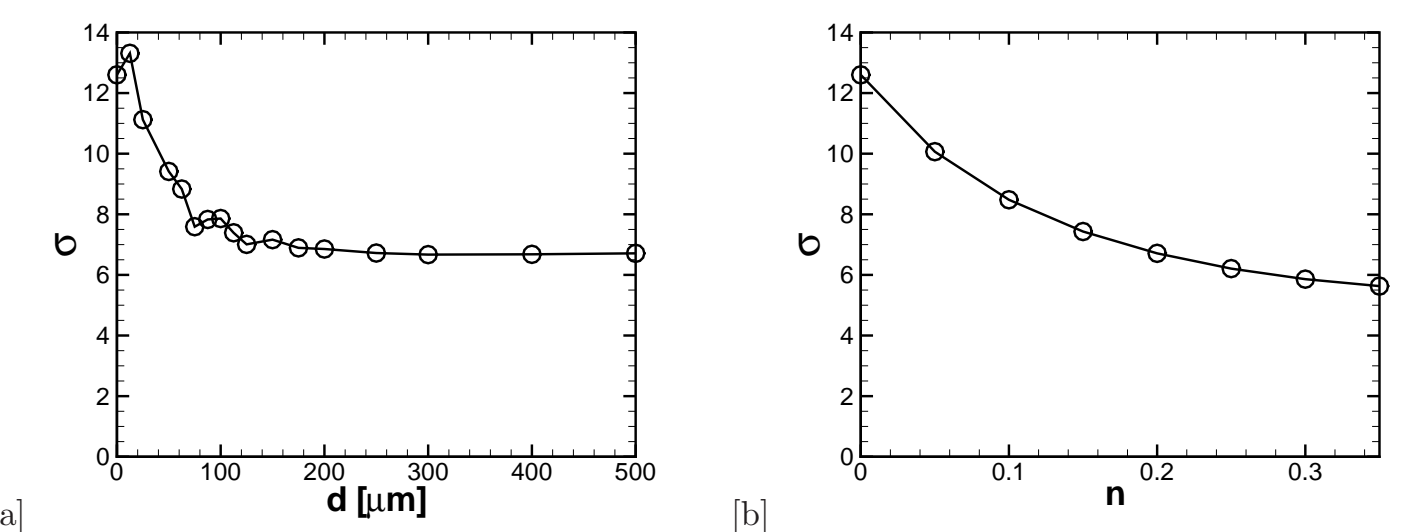

Figure 9. Cone case: (a) Maximal growth rate against pore depth $(n=0.2$ and $r=0.25 \mu m$ ) (b) Maximum growth rate against porosity $(r=0.25 \mu m)$

\section{Conclusion}

A second mode stability analysis was performed for a boundary layer flow at Mach 6 over a smooth wall and various porous walls. The influence of the porosity, the radius and the thickness of the pores were investigated. For this parameter study three different codes are used: The linear stability code SLST of the University of Southampton in comparison with the DLR linear stability code NOLOT and finally direct numerical simulations including the modelling of the pores inside the wall. Excellent agreements of all three codes were demonstrated for the smooth wall case. The parameter study shows for the porous walls small differences between LST and DNS as demonstrated in former studies. These are in an acceptable range for two completely different methods. Added to the different approaches the modelling of the pore shapes are different. Both LST codes show an excellent agreement for all porous wall cases. The differences are limited on the $5^{\text {th }}$ decimal of the growth rate. Finally a preview of planned wind tunnel tests without and with porous surfaces has been given.

\section{Acknowledgements}

The presented results are partly performed in the framework of the IMENS-3C project. Thus the authors would like to acknowledge the support of the IMENS-3C project managment Dr. A. Gülhan and Dr. H.-D. Speckmann.

\section{References}

${ }^{1}$ Mack, L.M. : "Boundary layer linear stability theory". AGARD Special course on stability and transition of laminar flow, 1984 .

${ }^{2}$ Malmuth, N.D., Fedorov, A.V., Shalaev, V., Cloe, J.D., Kholkhlov, A.: "Problems in High Speed Flow Prediction Relevant to Control". AIAA Paper 98-2995, June 1998.

${ }^{3}$ Fedorov, A.V, Malmuth, N.D., Rasheed, A., Hornung H.G.: "Stabilization of hypersonic boundary layers by porous coatings". AIAA Journal Vol. 39, No. 4, pp. 605-610, 2001.

${ }^{4}$ Rasheed, A., Hornung, H.G., Fedorov, A.V., Malmuth, N.D.: "Experiments on passive hypervelocity boundary-layer control using an ultrasonically absorptive surface". AIAA Journal, Vol. 40, No. 3, pp. 481-489, 2002.

${ }^{5}$ Maslov, A.A.: "Experimental and theoretical studies of hypersonic laminar flow control using ultrasonically absorptive coatings (UAC)". Report ISTC 2172-2001, May 2003.

${ }^{6}$ Bres, G.A., Colonius, T., Fedorov, A.V.: "Interaction of Acoustic Disturbances with Micro-Cavities for Ultrasonic Absorptive Coatings". AIAA 2008-3903, 5th AIAA Theoretical Fluid Mechanics Conference, 23 - 26 June 2008, Seattle, Washington.

${ }^{7}$ Bres, G.A., Colonius, T., Fedorov, A.V.: "Stability of Temporally-Evolving Supersonic Boundary Layers over MicroCavities for Ultrasonic Absorptive Coatings". AIAA 2008-4337, 5th AIAA Theoretical Fluid Mechanics Conference, 23 - 26 June 2008, Seattle, Washington. 
${ }^{8}$ Sandham, N.D., Lüdeke, H. : "A numerical study of Mach 6 boundary layer stabilisation by means of a porous surface". 47th AIAA Aerospace Sciences Meeting, 5 - 8 January, 2009, Orlando, Florida.

${ }^{9}$ Lüdeke, H., Sandham, N.D., Wartemann, V., : "Numerical Investigation of Transition Control by Porous surfaces in Hypersonic Boundary Layers". 16th DGLR Symposium of Stab, 3 - 4 November 2008, Aachen, Germany.

${ }^{10}$ Hein, S., Bertolotti, F.P., Simen, M., Hanifi, A., Henningson, D.: "Linear nonlocal instability analysis - the linear NOLOT code". DLR-IB 223-94 A56, 1994.

${ }^{11}$ Enk, S.: "Ein Verfahren höherer Ordnung in FLOWer für LES". DLR IB-124-2007/8, Institut für Aerodynamik und Strömungstechnik, 2007, Braunschweig, Germany.

${ }^{12}$ Malik, M.R.: "Numerical methods for hypersonic boundary layer stability". Journal of Computational Physics, Vol.86, No. 2, pp. 376-413, 1990.

${ }^{13}$ Fedorov, A., Shiplyuk, A., Maslov, A., Burov, E. and Malmuth, N.: "Stabilization of a hypersonic boundary layer using an ultrasonically absoptive coating", J. Fluid Mech. 479, pp. 99-124, 2003.

\section{A. Compressible Navier-Stokes equations}

The compressible Navier-Stokes equations for a flow of a perfect gas with density $\rho$, velocity components $u_{i}$, pressure $p$ and internal energy $e$, can be written in conservative or primitive form. The conservative formulation is:

$$
\begin{gathered}
\frac{\partial \rho}{\partial t}+\frac{\partial \rho u_{j}}{\partial x_{j}}=0 \\
\frac{\partial \rho u_{i}}{\partial t}+\frac{\partial \rho u_{i} u_{j}}{\partial x_{j}}+\frac{\partial p}{\partial x_{i}}=\frac{\partial \tau_{i j}}{\partial x_{j}}+f_{i} \\
\frac{\partial \rho E}{\partial t}+\frac{\partial(\rho E+p) u_{i}}{\partial x_{i}}=-\frac{\partial q_{i}}{\partial x_{i}}+\frac{\partial u_{i} \tau_{i j}}{\partial x_{j}}+g,
\end{gathered}
$$

where $E=e+u_{i} u_{i} / 2$. An equivalent set of equations for primitive variables may be derived by subtracting $u_{i}$ times equation (11) from (12) and subtracting $u_{i}$ times the resulting momentum equation and $e$ times (11) from (13). This leads to following primitive formulation of the Navier-Stokes equations:

$$
\begin{gathered}
\frac{\partial \rho}{\partial t}+u_{j} \frac{\partial \rho}{\partial x_{j}}+\rho \frac{\partial u_{j}}{\partial x_{j}}=0 \\
\rho \frac{\partial u_{i}}{\partial t}+\rho u_{j} \frac{\partial u_{i}}{\partial x_{j}}+\frac{\partial p}{\partial x_{i}}=\frac{\partial \tau_{i j}}{\partial x_{j}}+f_{i} \\
\rho \frac{\partial e}{\partial t}+\rho u_{j} \frac{\partial e}{\partial x_{j}}+p \frac{\partial u_{i}}{\partial x_{i}}=-\frac{\partial q_{i}}{\partial x_{i}}+\tau_{i j} \frac{\partial u_{i}}{\partial x_{j}}+g-u_{i} f_{i} .
\end{gathered}
$$

